Reprod. Nutr. Dévelop., 1982, 22 (1 B), 275-282.

\title{
Innervation motrice de la souris Trembler
}

\author{
J. L. LEFAIX $\left({ }^{1}, 2\right)$, H. L. KOENIG $\left({ }^{2}\right)$, M. VIGNY $\left({ }^{3}\right)$, J.-M. BOURRE $\left({ }^{4}\right)$
}

(1) Laboratoire de Radiobiologie appliquée, CNRZ, 78350 Jouy-en-Josas.

(2) Université P. et M. Curie, Laboratoire de Cytologie, 75230 Paris.

(3) Ecole Normale Supérieure, Laboratoire de Neurobiologie, 75005 Paris.

(4) INSERM U 134, Hôpital de la Salpêtrière, 75651 Paris.

\section{Summary. Trembler mouse motor innervation.}

The effects on muscle fibres and particularly on their motor end-plates, by axons whose Schwann cells fail to form and maintain a normal myelin sheath, were examined by morphological and biochemical techniques. In the soleus (slow type), the innervation area was more extensive along the muscle fibre; the most frequent modifications were : abnormal axonal branching pattern, swellings of the terminal branches and terminal and preterminal sprouting. Additional clusters of $\mathrm{ACh}$ receptors were frequent and $\mathrm{AChE}$ activity was unaffected. In the fast part of the gastrocnemius, neuromuscular junctions were slightly affected : sprouting was slight and all the muscle fibres had a single cluster of AChE receptors; AChE activity was modified.

In trembler mouse, the alteration of axon-Schwann cell relationships interacted with the regulatory processes ensuring the maintenance and stability of muscle fibre innervation.

\section{Introduction.}

La mutation murine Trembler, décrite en 1951 par Falconner, correspond à un caractère dominant porté par le chromosome 11 (Guénet, 1980). Elle est caractérisée par une déficience de la myélinisation dans le système nerveux périphérique. La gaine de myéline des axones est anormalement mince dans les nerfs périphériques, chez les animaux adultes (Ayers et Anderson, 1976 ; Low, 1976). En outre, ces axones présentent une succession de segments myélinisés et non-myélinisés et sont entourés, à l'exclusion des fibres de Remak, par des figures dites en " bulbe d'oignon " et par d'abondantes fibres de collagène (Ayers et Anderson, 1973). L'origine de la neuropathie, chez la souris Trembler, est purement schwannienne ; elle résulte de l'incapacité des cellules de Schwann à former et à maintenir une gaine de myéline normale (Aguayo et al., 1977, 1979).

Récemment, nous avons montré que les anomalies de myélinisation des nerfs moteurs périphériques étaient accompagnées par des modifications considérables de l'innervation motrice des fibres musculaires (Koenig et al., 1980). Nos observations, confirmées par Duchen et al. (1980), indiquent que les modifications patho- 
logiques sont plus importantes dans les muscles à fibres de type lent que dans ceux de type rapide.

II nous a donc paru intéressant d'effectuer une analyse morphométrique comparée de l'innervation motrice dans le muscle soléaire, à fibres de type lent et dans la partie superficielle du muscle gastrocnémien où prédominent des fibres de type rapide (Duchen, 1970), chez les souris Trembler et témoins.

\section{Matériel et méthodes.}

Les muscles soléaire et gastrocnémien (partie " rapide ») ont été prélevés chez 37 souris Trembler (B6D2Tr) et 37 témoins (B6D2 +) adultes et traités pour la mise en évidence des marqueurs morphologiques et biochimiques des synapses neuromusculaires (Koenig, 1979). Les terminaisons nerveuses motrices ont été imprégnées par des sels d'argent (méthode de Bielschowsky, modifiée par Gros et Schultze, 1918). Les gouttières synaptiques des plaques motrices ont été visualisées par la mise en évidence de leur activité cholinestérasique (méthode de Koelle et Friedenwald, modifiée par Couteaux et Taxi, 1952). Les agrégats de récepteurs à l'acétylcholine ( $\mathrm{R}-\mathrm{ACh}$ ) ont été révélés par radioautographie après incubation

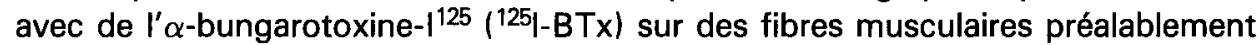
isolées par dissociation ( $\mathrm{J}$. Powell, technique non publiée). Chaque muscle a été traité par deux des techniques indiquées ci-dessus. L'activité spécifique de l'acétylcholinestérase a été mesurée, biochimiquement, par la méthode d'Ellman et al. (1961) et les formes moléculaies de cette enzyme ont été séparées par ultracentrifugation en gradient de saccharose (Bon et al., 1979).

L'analyse morphométrique a été effectuée à l'aide d'un micromètre oculaire pour mesurer la longueur : 1) des zones d'innervation, sur des coupes à congélation de 40 à $50 \mu \mathrm{m}$ d'épaisseur ; 2) des gouttières synaptiques, sur des fibres musculaires dissociées et 3 ) des agrégats de R-ACh, sur des fibres isolées. Le nombre et la longueur totale de rameaux des arborisations terminales ont été mesurés par projection des préparations sur l'écran d'un analyseur d'images Kontron (MOP), à l'aide d'une chambre claire. L'extrême polymorphisme des anomalies de l'innervation motrice des fibres d'un même muscle, que l'on observe après les imprégnations argentiques (Koenig et al., 1980), nous a incité à effectuer l'analyse morphométrique sur les seules fibres qui ne présentent qu'une seule place motrice dont l'aspect est comparable au témoin.

\section{Résultats.}

1. Terminaisons nerveuses motrices. - Dans les muscles que nous avons examinés, les plaques motrices sont toutes remaniées. Le diamètre des rameaux terminaux est irrégulier (fig. 2 et 4 ) ; de nombreux " empâtements 》 sont observés, sur un même rameau ou, plus fréquemment, aux points de bifurcation des rameaux (fig. 2, 3 et 4). La disposition spatiale de la ramure terminale, relativement semblable dans le Gastrocnémien (partie rapide) à celle du témoin (fig. 1 et 2), est le plus souvent désorganisée dans le Soléaire (fig. 3 et 4). L'ensemble de 
ces modifications sont observées pour les fibres qui ont une innervation « simple », à plaque motrice unique (90 p. 100 pour le Gastrocnémien et 60 p. 100 pour le Soléaire ( $\left.{ }^{1}\right)$ ) et pour les fibres dont l'innervation est « complexe ". II s'agit alors de fibres qui présentent plusieurs arborisations terminales (fig. 4), de fibres à innervation géante ou de fibres qui reçoivent des rameaux résultant d'un " sprouting » préterminal ou terminal (Koenig et al., 1980).

Dans le muscle gastrocnémien (partie rapide), le diamètre des fibres musculaires et la longueur des zones d'innervation (de 14,4 à $38,4 \mu \mathrm{m}$ ) sont similaires chez le Trembler et le témoin (tabl. 1). Toutefois, le nombre et la longueur totale des rameaux terminaux subissent un accroissement faible, mais significatif (environ 35 p. $100 ; p<0,01$; tabl. 1). En comparaison, les modifications de l'innervation motrice dans le muscle soléaire du mutant sont quantitativement plus importantes. Alors que les fibres ont un diamètre identique chez le Trembler et le témoin, la longueur des zones d'innervation varie de 19,2 à 43,2 $\mu \mathrm{m}$ chez le témoin et de

\section{TABLEAU 1}

Analyse morphométrique : muscle gastrocnémien (partie rapide)

\begin{tabular}{|c|c|c|c|}
\hline Mesures effectuées (exprimées en $\mu \mathrm{m}$ ) & Trembler & Témoin & $\begin{array}{l}\text { Rapport } \\
\mathrm{Tr} / \mathrm{Te}\end{array}$ \\
\hline Diamètre des fibres musculaires $\left({ }^{2}\right)$. & $\underset{(171)}{45,7 \pm 9,5}$ & $46,0 \underset{(51)}{ \pm} 8,4$ & 0,99 \\
\hline Longueur des gouttières synaptiques $\left({ }^{2}\right)$ & $\underset{(171)}{34,3 \pm 7,2}$ & $31,3 \pm(51)$ & 1,1 \\
\hline Longueur de la zone d'innervation (1) ........ & $\underset{(140)}{26,6 \pm} 5$ & $28,0 \pm(47) 5,7$ & 0,95 \\
\hline Longueur des rameaux terminaux (1). & $\begin{array}{c}101,8 \pm 27,4 \\
(140)\end{array}$ & $74,4 \underset{(47)}{ \pm 20,1}$ & 1,36 \\
\hline $\begin{array}{c}\text { Nombre de rameaux des arborisations ter- } \\
\text { minales }(1) \ldots \ldots \ldots \ldots \ldots \ldots \ldots \ldots \ldots \ldots \ldots \ldots\end{array}$ & $\underset{(140)}{5,4 \pm} 1,2$ & $4,0 \pm \underset{(47)}{ \pm} 1$ & 1,35 \\
\hline Longueur des agrégats de $\mathrm{R}-\mathrm{ACh}(3)$. & $\begin{array}{l}31,3 \pm \\
(114)\end{array}$ & $32,7 \underset{(52)}{ \pm} 4,0$ & 0,95 \\
\hline
\end{tabular}

Les chiffres correspondent, pour chaque cas, à la moyenne des mesures $( \pm$ déviation standard) effectuées sur toutes les fibres examinées (nombres entre parenthèses).

(1) Mesurée sur des coupes de 40-50 $\mu \mathrm{m}$ imprégnées par des sels d'argent ; (2) mesurée sur des fibres dissociées après visualisation de l'activité AChEsique ; $\left({ }^{3}\right)$ mesurée sur des radioautographies de fibres isolées après incubation avec 125I-BTX.

(1) 830 fibres du Gastrocnémien et 1290 du Soléaire ont été examinées. 

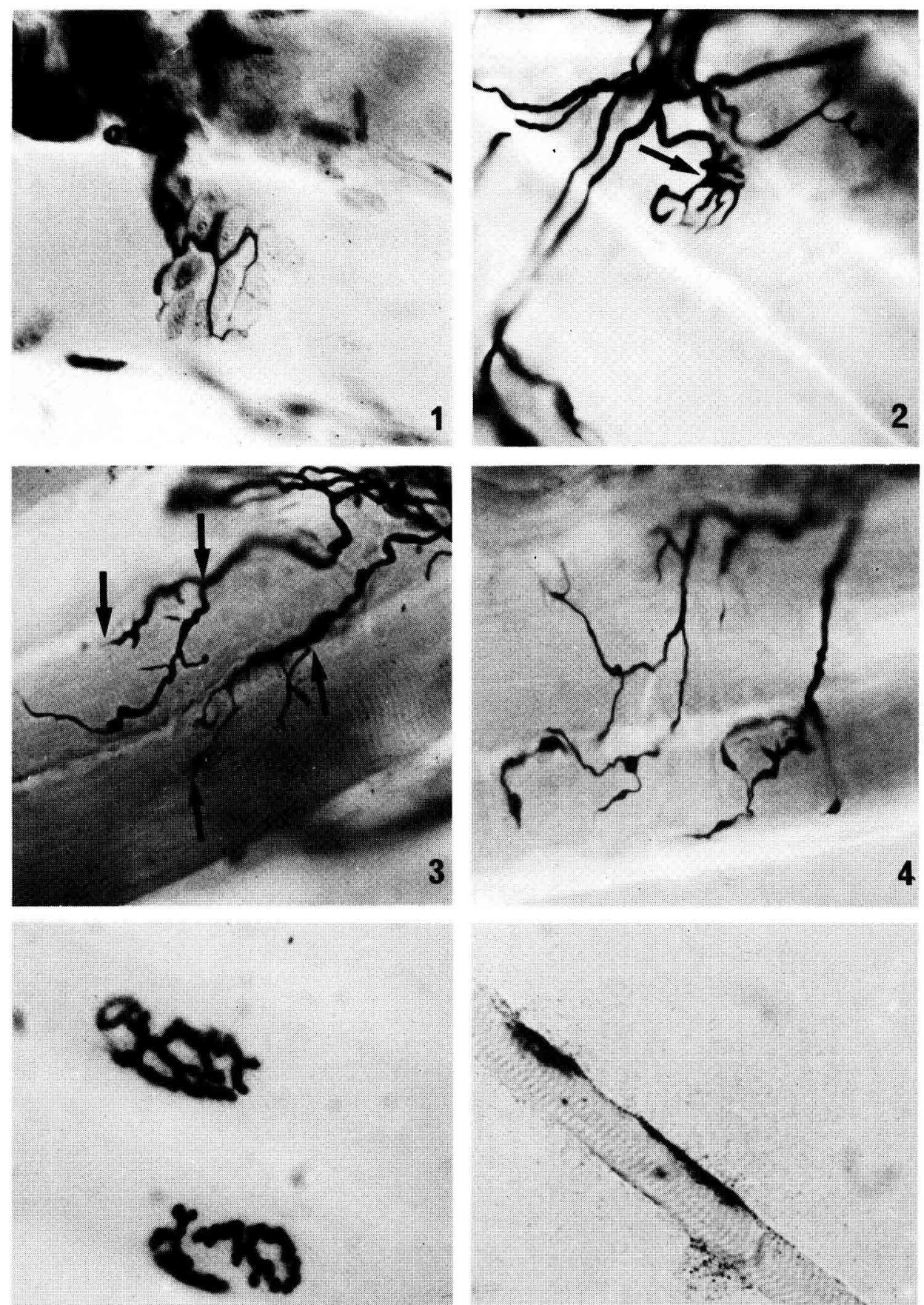

5

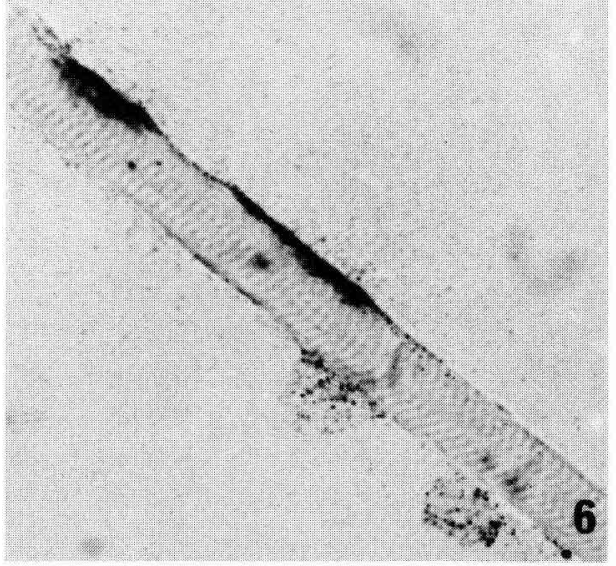


33,6 à 81,6 $\mu \mathrm{m}$ chez le Trembler. Le nombre des rameaux et leur longueur totale sont augmentés d'environ 2 fois ( $p<0,01$; tabl. 2 ).

2. Gouttières synaptiques. - La détection histochimique de l'activité cholinestérasique des plaques motrices, qui permet de visualiser les gouttières synaptiques, montre que dans les 2 muscles du Trembler examinés, on ne trouve à de rares exceptions près, qu'une seule plaque motrice par fibre musculaire. Dans le muscle gastrocnémien du mutant, les gouttières synaptiques ont l'aspect en couronne qui caractérise les plaques motrices des Mammifères. Par contre, dans le muscle soléaire les gouttières sont généralement plus longues que chez le témoin, en moyenne de 40 p. 100 (fig. 5 ; tabl. 2).

3. Récepteurs à l'acétylcholine. - Chez le Trembler, toutes les fibres du muscle gastrocnémien présentent un seul agrégat (cluster) de récepteurs à l'acétylcholine, dont les longueurs ne sont pas significativement différentes de celles mesurées dans le muscle témoin (tabl. 1). Dans le muscle soléaire, $60 \mathrm{p}$. 100 des fibres seulement ont un agrégat unique de R-ACh ; les longueurs de ces agrégats sont, en moyenne, supérieures de 40 p. 100 à ceux du muscle témoin ( $p<0,01$; tabl. 2). Sur les autres fibres du muscle, on dénombre de 1 à 4 agrégats additionnels, séparés par des distances variables ; leur longueur totale est, en moyenne, multipliée par un facteur 2 (fig. 6 ; tabl. 2).

4. Activité AChE et formes moléculaires. - Chez les souris Trembler et témoins, l'activité spécifique de l'acétylcholinestérase est pratiquement identique dans les muscles soléaires et gastrocnémien (celui-ci étant prélevé en totalité) ; de

FIG. 1. - Plaque motrice : muscle gastrocnémien de souris témoin. La forme ramassée de l'arborisation terminale est caractéristique des jonctions neuromusculaires chez les mammifères. Méthode de Bielschowsky-Gros (gross. $\times 600$ ).

FIG. 2. - Terminaison nerveuse : muscle gastrocnémien de souris Trembler. Observer le remaniement de sa morphologie, le grand nombre de rameaux et les épaississements caractéristiques (flèche). Méthode de Bielschowsky-Gros (gross. $\times 360$ ).

FIG. 3. - Terminaison nerveuse : muscle soléaire de souris Trembler. On note un allongement de l'arborisation terminale et une disposition inhabituelle des rameaux. La longueur de la zone d'innervation a été mesurée entre les flèches. Méthode de Bielschowsky-Gros (gross. $\times 360$ ).

FIG. 4. - Terminaisons nerveuse " complexes" : muscle soléaire de souris Tremb/er. La fibre musculaire du bas présente deux plaques motrices. Méthode de Bielschowsky-Gros (gross. $\times 360)$.

FIG. 5. - Gouttières sous-neurales: muscle soléaire de souris Trembler. Activité AChE (méthode de Koelle) (gross. $\times$ 430). Les deux plaques motrices sont plus allongées que chez le témoin (voir tabl. 2).

FIG. 6. - Agrégats de récepteurs à l'acétylcholine: muscle soléaire de souris Trembler. Radioautographie après incubation avec |'125|-BTx (gross. $\times 230$ ). La fibre musculaire présente deux agrégats, celui de gauche a une longueur voisine de celle des témoins, celui de droite est caractéristique de l'allongement observé chez le Trembler. 
même le pourcentage des formes asymétriques de l'enzyme (A $8+A$ 12) ne change pas dans le soléaire du mutant. Par contre, comparée au témoin, l'activité des formes A 8 et A 12 est augmentée d'environ 45 p. 100 dans le muscle gastrocnémien du Trembler.

TABLEAU 2

Analyse morphométrique : muscle soléaire

\begin{tabular}{|c|c|c|c|}
\hline Mesures effectuées (exprimées en $\mu \mathrm{m}$ ) & Trembler & Témoin & $\begin{array}{l}\text { Rapport } \\
\mathrm{Tr} / \mathrm{Te}\end{array}$ \\
\hline Diamètre des fibres musculaires $(2)$ & $\underset{(161)}{35,6 \pm} 9$ & $33,4 \underset{(50)}{ \pm} 7,6$ & 1,06 \\
\hline Longueur des gouttières synaptiques $(2)$ & $\begin{array}{l}50,4 \pm 11,7 \\
(161)\end{array}$ & $33,9 \pm \underset{(50)}{ \pm} 7,3$ & 1,48 \\
\hline Longueur de la zone d'innervation (1). & $\begin{array}{l}54,0 \pm 10,1 \\
(106)\end{array}$ & $27,8 \underset{(56)}{ \pm} 7,1$ & 1,94 \\
\hline Longueur des rameaux terminaux (1) & $\begin{array}{l}203,4 \pm 54,0 \\
(106)\end{array}$ & $93,9 \underset{(56)}{ \pm} 25,7$ & 2,16 \\
\hline $\begin{array}{c}\text { Nombre de rameaux des arborisations ter- } \\
\text { minales }(1) \ldots \ldots \ldots \ldots \ldots \ldots \ldots \ldots \ldots \ldots \ldots \ldots\end{array}$ & $\underset{(106)}{8,8 \pm} 2,4$ & $3,7 \underset{(56)}{ \pm} 1,3$ & 2,37 \\
\hline Longueur des agrégats uniques de R-ACh $\left({ }^{3}\right)$.. & $44,6 \underset{(95)}{ \pm} 17$ & $32,7 \underset{(54)}{ \pm} 8,34$ & 1,36 \\
\hline $\begin{array}{l}\text { Longueur totale des agrégats multiples de } \\
\text { R-ACh (3) } \ldots \ldots \ldots \ldots \ldots \ldots \ldots \ldots \ldots \ldots \ldots \ldots\end{array}$ & $\begin{array}{r}66,52 \\
(64)\end{array}$ & ------ & \\
\hline
\end{tabular}

Les chiffres correspondent, pour chaque cas, à la moyenne des mesures ( \pm déviation standard) effectuées sur toutes les fibres examinées (nombres entre parenthèses).

(1) Mesurée sur des coupes de 40-50 $\mu \mathrm{m}$ imprégnées par des sels d'argent; (2) mesurée sur des fibres dissociées après visualisation de l'activité AChEsique ; ${ }^{3}$ ) mesurée sur des radioautographies de fibres isolées après incubation avec ${ }^{125}$-BTx.

\section{Conclusions et discussion.}

Les résultats quantitatifs que nous avons obtenus confirment que les relations nerf-muscle dépendent d'interactions complexes dans lesquelles la nature des fibres musculaires, comme l'axone moteur, jouent un rôle important.

Dans le muscle gastrocnémien du Trembler, la pousse axonale anarchique, que I'on observe dans le soléaire (sprouting terminal et pré-terminal, multiinnervation), est rare. Dans la majorité des fibres de ce muscle, la surface synaptique (déterminée par la mesure des 3 marqueurs morphologiques : zone d'innervation motrice, gouttières synaptiques et agrégats de R-ACh) n'est pas modifiée (tabl. 1). L'accroissement, dans des proportions comparables, de la longueur de la 
ramure terminale et des formes asymétriques de l'AChE, suggère que ces formes seraient, non seulement induites par les neurones (Vigny et al., 1976 ; Koenig, 1979), mais que leur taux serait directement lié a l'abondance des rameaux axoniques enchassés dans la gouttière synaptique.

Dans le muscle soléaire du Trembler, les ramures terminales des axones moteurs, pour les fibres à innervation unique, occupent une surface plus grande (longueurs doubles; tabl. 2) que la surface synaptique (augmentation d'environ 40 p. 100 des gouttières et des agrégats de R-ACh ; tabl. 2). Les rameaux excédentaires émis par l'axone, hors de la plaque motrice, ne semblent donc pas capables d'induire la formation des différenciations post-synaptiques (qui caractérisent les jonctions neuromusculaires), ni l'augmentation des formes asymétriques de l'AChE (rapport Trembler/Témoin = 1). Cette interprétation est corroborée par le fait que 40 p. 100 des fibres du soléaire présentent une innervation " complexe ", souvent multiple, alors que la présence de plusieurs gouttières sur la même fibre musculaire est exceptionnelle.

En conclusion, l'analyse comparée des muscles soléaire et gastrocnémien chez la souris Tremb/er indique : 1) que la formation de ramifications " complexes " des axones moteurs est liée a la nature des fibres qui constituent les muscles ; 2) que l'agrégation additionnelle de récepteurs a l'ACh est induite par les arborisations terminales excédentaires ; 3) que ces arborisations inhabituelles ne sont pas capables d'induire de nouvelles focalisations de l'AChE ni d'augmenter le taux de ses formes asymétriques; 4) que lorsque la ramure est accrue à l'intérieur des plaques motrices normales, elle détermine une augmentation proportionnelle des formes asymétriques de l'AChE.

7e Réunion du groupe Développement I.N.R.A., Nouzilly/Tours, 14-15 mai 1981.

Remerciements. - Ce travail a été partiellement réalisé grâce à un contrat INSERM (CRL n 806 006).

Nous exprimons nos remerciements à M. F. Lachapelle pour le soin apporté à l'élevage des souris et à Mme P. Cloup pour la réalisation de l'iconographie.

\section{Références}

AguAYo A. J., ATTIWELL M., TRECARTEN J., PERKINS S., BRAY G. M., 1977. Abnormal myelination in transplanted Trembler mouse Schwann cells. Nature, 265, 73-74.

AGUAYO A. J., BRAY G. M., PERKINS S., 1979. Axon-Schwann cell relationships in neuropathies of mutant mice. Ann. N. Y. Acad. Sci., 317, 512-531.

AYERS M. M., ANDERSON R. Mc D., 1973. Onion bulb neuropathy in the Trembler mouse : A model of hypertrophic interstitial neuropathy (Dejerine-Sottas) in Man. Acta neuropathol., 25. 54-70.

AYERS M. M., ANDERSON R. Mc D., 1976. Development of Onion bulb neuropathy in the Trembler mouse. Morphometric study. Acta neuropathol., 36, 137-152.

BON S., VIGNY M., MASSOULIÉ J., 1979. Asymetric and globular forms of acetylcholinesterase in mammals and birds. Proc. nat. Acad. Sci., 76, 2546-2550. 
COUTEAUX R., TAXI J., 1952. Recherches histochimiques sur la distribution des activités cholinestérasiques au niveau de la synapse myoneurale. Arch. Anat. Microsc. Morphol. expér., 41, 352-392.

DUCHEN L. W., 1970. Changes in motor innervation and cholinesterase localization induced by botulinum toxin in skeletal muscle of the mouse : differences between fast and slow muscles. J. Neurol. Neurosurg. Psychiat., 33, 40-54.

DUCHEN L. W., GALE A. N., GOMEZ S., 1980. Motor end-plate morphology and neuromuscular transmission in the mutant Trembler mouse. J. Physiol., 308, $18 \mathrm{P}$.

ELLMAN G. L., COURTNEY K. D., ANDRES V., FEATHERSTONE R. M., 1961. A new and rapid colorimetric determination of acetylcholinesterase activity. Biochem. Pharmacol., 7, 88-95.

FALCONNER D. S., 1951. Two mutants " Trembler " and " Reeler " with neurological actions in the house mouse (mus musculus L.). J. Genet., 50, 192-201.

GROS V., SCHULTZE O., 1918. Neues zur mikroskopischen Untersuchung des Zentralnervensystems. Sitzunġsb. Physikal. med. Gesellsch., Würzburg, 1-5.

GUÉNET J. L., 1980. Mutants of the mouse with an abnormal myelination : a review for geneticists, 11-21. In N. BAUMANN, Neurological mutations affecting myelination. INSERM, Symp. 14, Elsevier, Amsterdam.

KOENIG H. L., LEFAIX J. L., BOURRE J. M., 1980. Abnormal motorinnervation in Tremb/er mutant muscles, 537-542. In N. BAUMANN, Neurological mutations affecting myelination. INSERM, Symp. 14, Elsevier, Amsterdam.

KOENIG J., 1979. Formation and maturation of nerve-muscle contacts in cultured rat embryo cells. Biol. cell., 35, 147-152.

LOW P. A., 1976. Hereditary hypertrophic neuropathy in the Trembler mouse. J. neurol. Sci., 30 , 327-341 et 343-368.

VIGNY M., KOENIG J., RIEGER F., 1976. The motor end-plate specific form of acetylcholinesterase : appearance during embryogenisis and re-innervation of rat muscle. J. Neurochem., 27, 1247-1253. 